\title{
A Rare Case of Multiorgan Involvement of Rosai Dorfman Disease in an Elderly Female
}

\author{
Rakesh Digambar Waghmare ${ }^{1}$ \\ 'Department of Pulmonary Medicine, Byramjee Jeejeebhoy Government Medical college, Pune, India.
}

\begin{abstract}
Sinus histiocytosis with massive lymphadenopathy (SHML), Rosai-Dorfman Disease, is a rare histiocytic syndrome first described by Rosai and Dorfman, most frequently seen in children and young adults.The disease is more common in males and in individuals of African descent but rare in Asians. It is mainly characterized by painless bilateral cervical lymph node enlargement and is often associated with fever and leucocytosis. This case is being reported for its rarity in presentation in an elderly female with both generalized nodal as well as extranodal manifestations. Without the awareness about RDD, the diagnosis of RDD is unexpected especially in South East Asian Countries where certain lymphadenopathies such as tuberculosis, metastatic malignancies and lymphomas are common.
\end{abstract}

Keywords: emperipolesis; rosai-dorfman disease; sinus histiocytosis with massive lymphadenopathy.

\section{INTRODUCTION}

SHML or RDD is a rare but well defined, benign histiocytic proliferative disorder of unknown etiology. The first report of sinus histiocytosis with massive lymphadenopathy (SHML) was described in 1965, by Destombes as a lipid storage disorder developing after inflammation ${ }^{1}$ but it were Rosai and Dorfman in 1969 described the clinical syndrome for which they coined the term SHML. ${ }^{2}$ This condition generally affects children and young adult, more common in males, and in African heritage ${ }^{3}$ but rare in Asians. ${ }^{4}$

This case is being reported for its rarity in presentation in an elderly female with both generalized nodal as well as extranodal manifestations.

\section{CASE REPORT}

A 60 year old multiparous female patient, housewife, non-addict, not a known case of any illness came with complaints of bilateral neck swellings and swelling in the right axillary region since eight months, low grade fever intermittently and generalised weakness with no history of loss of weight, night sweats. She had received a course of antitubercular treatment from private practitioner for six months with no decrease in the size of swellings. On clinical examination she was averagely built and nourished with pallor along with bilateral multiple submental, submandibular, parotid and cervical lymph nodes with few were matted, ranging in size from $1-4 \mathrm{~cm}$, firm, freely mobile, non-tender. A $2 \times 2 \mathrm{~cm}$ well defined, firm, non-tender lymph node was present in the right axillary area. Rest of the physical examination was unremarkable. 
Investigations were-

$\mathrm{Hb}-8.7 \mathrm{gms} / \mathrm{dl}$, normocytic normochromic anaemia .TLC-15,500/mm³ ${ }^{3}$ DLC: N-80\% , L- $18 \%$, E-2\%. ESR was $95 \mathrm{~mm}$ at one hour. HIV and $\mathrm{HbsAg}$ negative. Other investigations were normal.

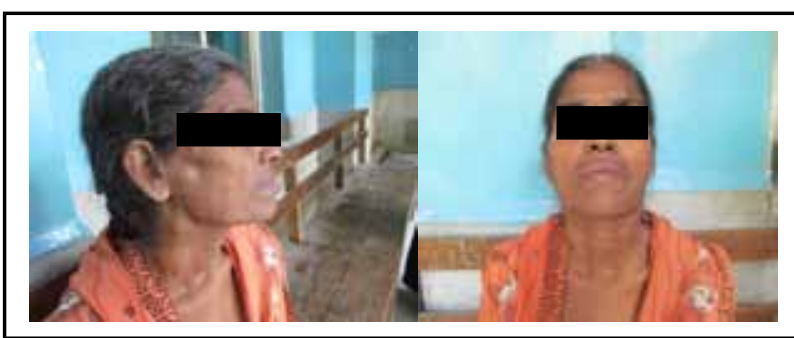

Figure 1. Photographs patient showing cervical lymphadenopathy.

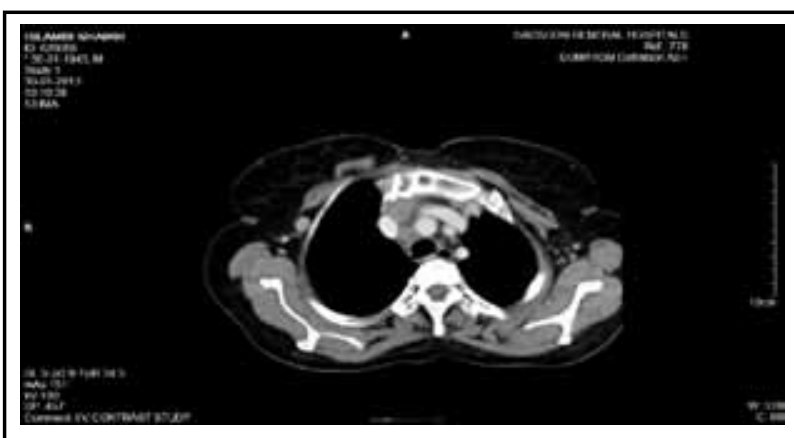

Figure 2. Ct chest showing mediastinal lymph nodes and osteolytic lesions in bones.

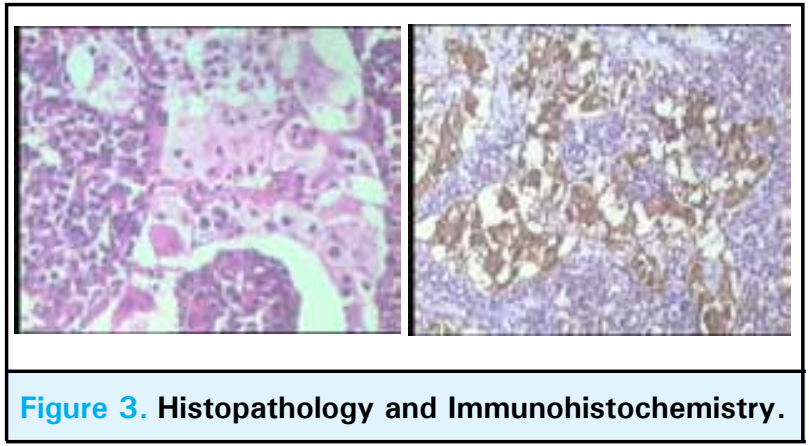

CT neck and thorax demonstrated intensely enhancing enlarged lymph nodes in Level I, II, III, IV, V, VII and bilateral parotid, left buccal largest measuring $2.2 \times 2 \mathrm{~cm}$ enlarged lymph nodes were also seen at prevascular, pretracheal, aortopulmonary and right hilar region. Multiple lytic lesions were noted in vertebral bodies, sternum, near right first sternochondral junction.

USG Abdomen- Extensive discrete and confluent lymphadenopathy in the periportal, peripancreatic regions and in the mesentry. Few of the lymph nodes were necrotic. Multiple well defined hypoechoic lesions were seen in the right lobe of liver which could represent histiocytic deposition.
FNAC of cervical lymph nodes showed multinucleated giant cells with inflammatory cells and was inconclusive.

Transbronchial needle aspiration cytology revealed numerous multinucleated giant cells with emperipolesis in the background of mixed inflammatory cells.

A lymph node biopsy was done which demonstrated dilated sinuses filled with histiocytes, few lymphocytes and plasma cells. Few of the histiocytes show emperipolesis. There was diffuse infiltration by plasma cells in the intersinusal tissue. Immunohistochemistry was positive for S-100. The presence of emperipolesis, or the engulfment of lymphocytes and erythrocytes by histiocytes that express $\mathrm{S}-100$, is considered diagnostic of RDD.

Patient was started on oral prednisolone $1 \mathrm{mg} / \mathrm{kg}$ with azathioprine and n-acetlycysteine. There was a regression in the size of lymph nodes and improved general condition within two months of starting treatment. Patient is currently on follow up with gradual tapering of steroids.

\section{DISCUSSION}

Since, its original description, Rosai-Dorfman disease has become a well-established clinicopathological entity. The most frequent clinical presentation of RDD is a massive bilateral and painless cervical lymphadenopathy with fever, night sweats and weight loss. This phenomenon has also been described as "bull neck". Mediastinal, inguinal \& axillary nodes may also be involved in $30-40 \%$ of patients. ${ }^{3}$ The retroperitoneum is an infrequent site of disease. ${ }^{5}$ These nodes may at times be matted and prominent by periportal fibrosis. Extranodal involvement by RDD has been documented in $43 \%$ of cases with the most frequent sites being skin, soft tissue, upper respiratory tract, multifocal bone, eye and retro-orbital tissue with lymphadenopathy or as an isolated initial manifestation of disease. ${ }^{3}$ Apparently, there is no relation between lymph node and extra nodal disease; they may even occur separately. ${ }^{6}$

Other reported sites include urogenital tract, breast, gastrointestinal tract, liver, pancreas and lungs, kidneys, thyroid, unifocal skeletal lesions. Skeletal lesions of RDD are typically osteolytic and can be confused radiographically with $\mathrm{LCH} .{ }^{3}$

Postulated causes include infectious causes like Herpesvirus 6 (HHV-6) and Epstein-Barr virus(EBV) immunodeficiency, autoimmune disease, and a neoplastic process; however, none has been substantiated. ${ }^{3}$

Our case was a 70 year old female who presented 
with generalized lymphadenopathy, fever, leukocytosis, and an elevated ESR with both nodal and extranodal involvement.

Histologically, lymph nodes show pericapsular fibrosis and dilated sinuses, heavily infiltrated with large histiocytes, lymphocytes and plasma cells. The presence of emperipolesis, or the engulfment of Iymphocytes and erythrocytes by histiocytes that express S-100, is considered diagnostic of RDD although not uniquely, ${ }^{3}$ were also present in our case.

Apart from S-100 antigen positivity, immunohistochemical stains of RDD cells are also positive for CD68, CD163, 41-antichymotrypsin, 41antitrypsin, fascin and HAM-56 while CD 1 a is typically negative. ${ }^{3}$

The clinical course is unpredictable with episodes of exacerbation and remissions that could last many years. The disease is often self-limiting with a very good outcome, complete spontaneous regression is known to occur, nevertheless $5-11 \%$ of patients die from their disease. The treatment modalities for RDD are nonspecific and include corticosteroids, chemotherapy with a combination of vinca alkaloids and alkylating agents, low dose interferon, antibiotic therapy, radiation therapy and surgical treatment with partial or total resection. ${ }^{7}$

In this case, patient was started on oral prednisolone with azathioprine and n-acetlycysteine. There was a regression in the size of lymph nodes with improved general condition within two months of starting treatment. Patient is currently on follow up with gradual tapering of steroids.

To conclude, Rosai-Dorfman disease should be considered in the differential diagnosis of granulomatous infection, pseudogranulomatous lesion and malignancy.RDD indeed is a rare finding, especially in Indian subcontinent. Physicians need to have a high degree of suspicion in patients presenting with generalized lymphadenopathy, with or without extranodal involvement, to diagnose RDD. Indolent course, presence of histiocytes with emperipolesis in inflammatory exudates and $\mathrm{S} 100$ positive immunohistochemical staining clinch the diagnosis in favour of Rosai Dorfman disease or sinus histiocytosis with massive lymphadenopathy.

\section{REFERENCES}

1. Destombes P. Adenitis avec surcharge lipidique, de $\mathrm{l}^{\prime}$ enfant ou de l'adultejeune, odservees aux Antilles et au Mali. Bulletin of the Society of Exotic Pathology. 1965;6:1169-75.

2. Rosai J, Dorfman RF. Sinus histiocytosis with massive lymphadenopathy: a newly recognized benign clinicopathological entity. Archives of Pathology, vol. 87, no. 1, pp. 63-70, 1969.

3. Abla, O. (2014). Rosai-Dorfman Disease. [online] Histiocytesociety.org. Available at: http://www. histiocytesociety.org/ document.doc?id=54 [Accessed 24 Aug. 2014].

4. Daniele Cristine Gomes Pinto, Tatiana de AguiarVidigal, Bruno de Castro,et all: Rosai-Dorfman disease in the differential diagnosis of cervical lymphadenopathy. Brazilian Journal of Otorhinolaryngology 2008;74(4): 632-5.
5. Marsh WL, Mc Carrick JP, Harlan DM. Sinus histiocytosis with massive lymphadenopathy. Occurrence in identical twins with retroperitoneal disease. Archives of Pathology and Laboratory Medicine 1988;112:298.

6. Carpenter RJ III, Banks PM, Mc Donald TJ, Sanderson DR. Sinus histiocytosis with massive Lynphadenopathy (Rosai-Dorfman disease):Report of a case with respiratory tract involvement. Laryngoscope 1978;88:1963-9.

7. Pulsoni A, Anghel G, Falcucci P, Matera R, Pescarmona E, Ribersani M, et al. Treatment of sinus histiocytosis with massive lymphadenopathy (Rosai-Dorfman disease): report of a case and literature review. American Journal Hematology2002; 69 (1): 67- 71. 